\title{
Diferencias de género en las universidades españolas y en sus órganos gerenciales*
}

\author{
López-Bonilla, Jesús Manuel** \\ Martínez-Torres, María del Rocío*** \\ Díaz-Fernández, María del Carmen ${ }^{\star \star \star \star}$
}

\section{Resumen}

Las universidades españolas son un reflejo de las desigualdades de género en la esfera pública. Diversos trabajos e informes publicados hasta la fecha muestran la falta de equidad en la participación de mujeres y hombres en las instituciones consideradas como democráticas. El objetivo principal de este trabajo es diagnosticar la composición de género en el ámbito de la gerencia universitaria española. Así, se analiza la presencia de la mujer en la universidad española, su representación en los órganos de gobierno y la evolución de estas cifras en los últimos años. Para ello, en primer lugar se revisa la normativa en materia de igualdad de género, tanto a nivel nacional como europeo y, en segundo lugar, se realiza un análisis longitudinal para abordar la situación de las mujeres y los hombres en las universidades españolas, tanto públicas como privadas. La investigación se cataloga como descriptiva. Los resultados reflejan una aparente tendencia a la igualdad formal en el acceso discente y docente a la universidad, pero esconde una segregación que sigue manteniendo a las mujeres en situaciones de desigualdad ante los hombres.

Palabras clave: Género, órganos de gobierno, representación paritaria, gerencia universitaria, España.

\section{Recibido: 29-03-12. Aceptado: 30-07-13}

* Este estudio ha sido financiado por el Ministerio de Educación y Ciencia (Proyecto de investigación EA2009-0039: Análisis del acceso de la mujer a los puestos directivos de las universidades españolas y propuestas para conseguir la igualdad participativa de género).

** PhD, Profesor Titular de Universidad, Facultad de Turismo y Finanzas, Universidad de Sevilla (España). E-mail: lopezbon@us.es

*** PhD, Profesora Titular de Universidad, Facultad de Turismo y Finanzas, Universidad de Sevilla (España). E-mail: rmtorres@us.es

**** PhD, Profesora Contratada Doctora, Facultad de Turismo y Finanzas, Universidad de Sevilla (España).E-mail: cardiaz@us.es 


\title{
Gender Differences at Spanish Universities and in their Managerial Organizations
}

\begin{abstract}
Spanish public universities are a reflection of gender inequalities in the public sphere. Various studies and reports published to date demonstrate a lack of equity in the participation of women and men in institutions that are considered democratic. The principle objective of this work is to diagnose gender composition in the Spanish university sphere; thus, the presence of women in Spanish universities, their representation in governing organisms and the evolution of these figures in recent years are analyzed. First, laws regarding matters of gender equality on the national and European levels are reviewed; second, a longitudinal analysis is made to approach the situation of women and men in both public and private Spanish universities. The research is catalogued as descriptive. Results reflect an apparent tendency to formal equality in learner and teacher access to the university, but a concealed segregation continues to keep women in situations of inequality with regard to men.
\end{abstract}

Key words: Gender, government organizations, joint representation, university management, Spain.

\section{Introducción}

Los cambios sociales de los últimos años con relación a la ruptura dicotómica entre el espacio público y privado, y la creciente complejidad del "pluralismo cultural" en un mundo globalizado, sitúan la necesidad de valorar la diversidad y su gestión estratégica como un elemento clave dentro de las organizaciones. La presencia de las mujeres en la vida pública obliga a las instituciones a replantear su cultura organizativa y sus políticas con base en una gestión de la diversidad para optimizar el aprovechamiento de los recursos y del potencial humano de esa "otra mitad de la población" que hasta hace poco permanecía invisible. Ante un mundo cada vez más globalizado, el aumento del potencial humano que representan las mujeres será un elemento clave para mejorar la competitividad de las organizaciones (Martínez et al, 2010).
La perspectiva de género permite visibilizar y explicar situaciones de inequidad y desigualdad entre mujeres y hombres, las que se asientan en relaciones de poder asimétricas entre los sexos, construidas socio-culturalmente en el proceso de socialización de género (González, 2004). Como indican Rico y Gómez-Limón (2011), no existe fundamento científico para la normalización de unas diferencias de género que son esencialmente sociales y culturales, históricamente constituidas desde los distintos roles que han cumplido mujeres y hombres y desde la división sexual del trabajo. En este sentido, esta división del trabajo condiciona, de forma significativa, el acceso de las mujeres a puestos de poder y restringe, por tanto, las posibilidades de desempeñar cargos directivos (García, 2007).

La Unión Europea, desde los años 90 , ha desarrollado políticas de igualdad 
en todos los ámbitos. En 1998, tres años después de la Conferencia Mundial de la Mujer celebrada en Pekín, la Dirección General de Investigación de la Unión Europea crea un grupo de trabajo encargado de analizar la situación de las mujeres en la ciencia y la tecnología de diversos países miembros, cuyas conclusiones se presentan en el Informe de la European Technology Assessment Network (ETAN, 2000). Entre ellas destaca el apartado cuyo subtítulo es "Promover la excelencia mediante la integración de la igualdad entre género" (Informe ETAN, 2000), en él se indica que la infra-representación de las mujeres amenaza los objetivos científicos de alcanzar la excelencia, además de ser un derroche y una injusticia.

La elaboración de informes sobre la desigual representación de mujeres y hombres en las instituciones, sobre las relaciones de poder que subyacen en las relaciones de género y sobre la necesidad de políticas de igualdad para un adecuado funcionamiento democrático es abundante. La igualdad de oportunidades entre mujeres y hombres, y su presencia equilibrada en los ámbitos de poder es una herramienta clave y una condición necesaria para avanzar en la democracia paritaria. Sin embargo, mientras las mujeres tengan invisibilidad, la representación política como elemento funcional de las instituciones democráticas nunca podrá ser equilibrada, ni el sistema democrático podrá ser justo.

Ante estos cambios sociales y políticos, el objetivo de este estudio es diagnosticar la composición de género en la universidad española, desde una perspectiva comparativa en el transcurso de más de una década y dentro del nuevo marco normativo que establece la obligación de alcanzar una representación paritaria en los órganos de toma de decisiones. Por ello, se realiza un análisis descriptivo donde se contempla tanto la presencia de mujeres y hombres en los distintos colectivos universitarios como su representación en los órganos de gobierno unipersonales y colegiados de las universidades españolas.

En el presente trabajo se llevó a cabo un triple análisis cuantitativo. En el primero de ellos se estudia la representación demográfica por género y se analizan las cifras desagregadas de la presencia de mujeres y hombres en todos los sectores universitarios: Personal Docente e Investigador (PDI), Personal de Administración y Servicios (PAS) y alumnado. El segundo análisis estudia la representación política, contrastando la composición por género de los órganos de gobierno universitarios. Paralelamente, en tercer lugar, se lleva a cabo un análisis longitudinal considerando como punto de referencia el estudio realizado por López y Martínez (1999), centrado en el análisis de género en los órganos de gobierno universitario durante el curso 1996-1997.

Aunque la condición de la mujer en el mercado de trabajo ha sido ampliamente abordada por estudios de género, como indican Iranzo y Richter (2002), pocas son las investigaciones realizadas hasta la fecha sobre las diferencias de género en los cargos de gestión universitaria en España. En la última década se han publicado algunos trabajos relevantes sobre las diferencias de género en la gestión de las universidades españolas, como son los de López y Martínez (1999); Hernández et al (2004); Rodríguez (2006); Tomás y Guilla- 
món (2009) y Tomas et al (2009). Todos estos estudios coinciden en valorar la escasa presencia de la mujer en el desempeño de tareas profesionales y cargos de gestión en el ámbito universitario. También se han realizado estudios similares en otros países obteniendo resultados similares. Así por ejemplo, White et al (2010) desarrollaron una investigación en ocho países, encontrando que la representación de las mujeres en cargos de gestión universitaria es muy reducida.

En el presente estudio, las principales fuentes de información fueron los datos de la Estadística de Enseñanza Superior del Instituto Nacional de Estadística para los cursos 1996-1997 y 2008-2009, así como de las propias instituciones educativas. La muestra que conforma este estudio estuvo constituida por la totalidad de las universidades que comprende la Educación Superior en España en 2009: 72 universidades españolas, de las que hay 46 universidades públicas de carácter presencial, dos universidades públicas de formación a distancia, 24 universidades privadas, siendo una de estas últimas una universidad de educación a distancia.

\section{Estrategias para la igualdad de género}

La realización de este estudio se deriva de la necesidad de mostrar los avances acontecidos en materia de representación de las mujeres en las Universidades como consecuencia de los cambios políticos y sociales de los últi- mos años. Adquiere su sentido, sobre todo, dentro del nuevo marco Europeo de Enseñanza Superior y en el contexto de un compromiso internacional de introducir la estrategia del mainstreaming en los países de la Unión Europea.

Desde la aprobación del Tratado de Roma en $1957^{1}$, los Estados Miembros se acogen a una legislación comprometida con la igualdad de trato entre mujeres y hombres. En dicho Tratado, se declara ilegal la discriminación laboral por razón de sexo. En 1995 se celebra la Conferencia Mundial de la Mujer en Pekín, en la que se acepta universalmente el mainstreaming, definido como "la integración sistemática de la igualdad de oportunidades entre hombres y mujeres en la organización y su cultura, en todos los programas, políticas y prácticas, y en las maneras de ver y de hacer las cosas" (Informe ETAN, 2000). Esta estrategia se introduce en la política europea en el año 1997 a través del Tratado de Amsterdam (Consejo de la Unión Europea, 1997), el cual regula toda discriminación basada en el sexo o la orientación sexual.

En 1999, en la "Conferencia Mujeres y Hombres", celebrada en París, los gobiernos de los Estados Europeos asumen el compromiso de avanzar en lo que se define como democracia paritaria. La Declaración Final de la Cumbre Europea, celebrada en Atenas en 1992, asigna el término de "democracia paritaria" a la presencia de mujeres y hombres en todos los ámbitos, sobre todo aquellos que dependen del Estado, en una proporción no 
inferior al $40 \%$ ni superior al $60 \%$ de cualquiera de los dos géneros. Por último, en 2004, el Consejo de Europa promulga la Directiva 2004/113/CE, mediante la cual, como se indica en su artículo 17, los Estados Miembros deben adoptar las disposiciones necesarias para garantizar el "principio de igualdad de trato en el acceso a bienes, servicios y suministros" antes del 21 de diciembre del año 2007.

Como consecuencia de estas recomendaciones, resoluciones y otras medidas específicas europeas, en España, las recientes medidas legislativas establecen un marco jurídico que obliga a las universidades públicas y privadas a tener presencia equilibrada de mujeres y hombres en sus órganos de gobierno. Dentro de este ámbito se establece el marco legal del que parte este estudio: la Ley Orgánica que modifica la Ley Orgánica de Universidades (LOMLOU), es decir, la Ley Orgánica 4/2007, de 12 de abril, por la que se modifica la Ley Orgánica 6/2001, de 21 de diciembre, de Universidades; y la Ley Orgánica 3/2007, de 22 de marzo de 2007, para la Igualdad efectiva de Mujeres y Hombres (Gobierno de España, 2007b).

Así pues, en el artículo 13 de la LOMLOU se dispone que "los estatutos establecerán las normas electorales aplicables, las cuales deberán propiciar en los órganos colegiados la presencia equilibrada entre mujeres y hombres". Una de las modificaciones importantes de esta Ley de Universidades es la creación de las Unidades de Igualdad y el seguimiento de la aplicación del principio de igualdad por parte de la Conferencia General de Política Universitaria.
En el artículo 16 de la Ley para la Igualdad efectiva de Mujeres y Hombres queda de manifiesto que "Ios Poderes Públicos procurarán atender el principio de presencia equilibrada de mujeres y hombres en los nombramientos y designaciones de los cargos de responsabilidad que les correspondan". Asimismo, la disposición adicional primera del Título VIII de dicha Ley (Gobierno de España, 2007a) especifica que "se entenderá por composición equilibrada la presencia de mujeres y hombres de forma que, en el conjunto a que se refiera, las personas de cada sexo no superen el sesenta por ciento ni sean menos del cuarenta por ciento".

Por último, en el artículo 23 de esta Ley (Gobierno de España, 2007a) se insta al sistema educativo a aplicar las medidas necesarias para el cumplimiento de la igualdad efectiva en todos los ámbitos, declarando que "el sistema educativo incluirá, dentro de sus principios de calidad, la eliminación de los obstáculos que dificultan la igualdad efectiva entre mujeres y hombres y el fomento de la igualdad plena de unas y otros".

Finalmente, el Plan Estratégico de Igualdad de Oportunidades 2008-2011 (Ministerio de Trabajo y Asuntos Sociales, 2007) introduce en sus Ejes las medidas dirigidas a garantizar la presencia equilibrada, mientras que las universidades, en sus estatutos internos, deben realizar las gestiones necesarias para garantizar el cumplimiento de la Ley e impulsar la aplicación de planes de igualdad donde se establezcan las áreas de actuación, objetivos y acciones necesarias para avanzar en el objetivo de igualdad de oportunidades entre mujeres y hombres. 


\section{Diferencias de género en las universidades españolas}

En este apartado se describe la representación demográfica y política por género en las universidades españolas.

\subsection{Representación demográfica por género en las universidades españolas}

En cuanto al alumnado, en los 12 años transcurridos entre los cursos 19961997 y 2008-2009 la población femenina presente en las universidades se incrementó de manera constante (Tabla 1). Del total del alumnado matriculado en 1996 , el $53,92 \%$ eran mujeres, mientras que en 2008 esta cifra alcanzó al $54,36 \%$. Si se centra la atención en las enseñanzas de primer y segundo ciclo universitario, las mujeres que iniciaron estudios durante el curso 1996-1997 suponían un 53,91\%, ascendiendo hasta el $55,65 \%$ en el curso 2008-2009. Otro dato significativo se encuentra en el alumnado femenino que finalizó sus estudios en ambos cursos extremos: un $58 \%$ en 1997, frente a un $61,4 \%$ en 2009 . Estos porcentajes sugieren un mayor índice de rendimiento aca- démico en las mujeres que en los hombres, que debería verse reflejado posteriormente en su carrera laboral.

En cuanto a los datos de la presencia de género por ramas de enseñanza, se comprueba que las mujeres siguen vinculadas a las áreas Sociales, Humanidades y Sanitarias (Tabla 2). Así, durante el período analizado, los datos de esta brecha de género apenas han cambiado, ya que en torno al $65 \%$ del alumnado matriculado en las áreas de Ciencias de la Salud, Humanidades y Ciencias Sociales y Jurídicas son mujeres, tanto en 1996 como en 2008. En la rama de Ciencias Experimentales es donde menos diferencias se produce, ya que en el curso 1996-1997 había un 52,7\% de mujeres con respecto a los hombres, y en 20082009 era de un $57,68 \%$. La mayor diferencia se encuentra en las enseñanzas técnicas, fuertemente masculinizadas, donde tan sólo el $25,5 \%$ del alumnado eran mujeres en $1996-1997$ y el $27,06 \%$ en 2008-2009.

La mayoría femenina en el alumnado universitario de primer y segundo ciclo se torna en un mayor equilibrio en cuanto a los estudios de tercer ciclo. Así, las mujeres y los hombres matriculados en los

\section{Tabla 1}

Representación demográfica por género en el alumnado de $1^{\circ}$ y $2^{\circ}$ ciclos universitarios

\begin{tabular}{lcccc}
\hline \multirow{2}{*}{ Alumnado } & \multicolumn{2}{c}{ Curso 1996-1997 } & \multicolumn{2}{c}{ Curso 2007-2008 } \\
\cline { 2 - 5 } & Mujeres (\%) & Hombres (\%) & Mujeres (\%) & Hombres (\%) \\
\hline Inicia estudios & 53,91 & 46,09 & 55,26 & 44,74 \\
Finaliza estudios & 58,00 & 42,00 & 60,91 & 39,09 \\
Matriculado & 53,92 & 47,08 & 54,65 & 45,35 \\
\hline
\end{tabular}

Fuente: Elaboración propia a partir de López y Martínez (1999) e INE (1998 y 2010). 


\section{Tabla 2}

\section{Representación demográfica por género en el alumnado de $1^{\circ}$ y $2^{\circ}$ ciclos por ramas de enseñanzas}

\begin{tabular}{lcccc}
\hline \multirow{2}{*}{ Estudios } & \multicolumn{2}{c}{ 1996-1997 } & \multicolumn{2}{c}{ 2008-2009 } \\
\cline { 2 - 5 } & Mujeres (\%) & Hombres (\%) & Mujeres (\%) & Hombres (\%) \\
\hline Ciencias Experimentales & 52,7 & 47,7 & 57,68 & 42,32 \\
Ciencias de la Salud & 70,38 & 29,62 & 73,30 & 26,70 \\
Ciencias Sociales y Jurídicas & 60,07 & 39,93 & 63,15 & 36,85 \\
Humanidades & 64,72 & 35,28 & 60,84 & 39,16 \\
Técnicas & 25,50 & 74,50 & 27,06 & 72,94 \\
\hline
\end{tabular}

Fuente: Elaboración propia a partir de López y Martínez (1999) e INE (1998 y 2010).

cursos de doctorado en el curso 19961997 suponían un $49,75 \%$ y un $50,25 \%$, respectivamente, ascendiendo al $51,46 \%$ en el caso de las mujeres en el curso 2008-2009. No obstante, lo más significativo es que las tesis doctorales aprobadas redujo ampliamente sus diferencias de género, pasando las tesis disertadas por mujeres de un $39,68 \%$ en el curso 1996 1997 a un $48,79 \%$ en el curso 2008-2009. Este es un dato muy relevante si se considera, como ya advertía Andreu (2002), que la discriminación por género en la universidad se produce principalmente a través de las tesis doctorales.

Centrándose en el ámbito laboral universitario, en cuanto al Personal Docente e Investigador (PDI) en las universidades públicas, la Tabla 3 muestra una subida de poco más de cuatro puntos porcentuales en la representación femenina, en contraste con los 18 puntos que alcanzan en las universidades privadas. Ciertamente, éstas últimas estaban más desequilibradas en el reparto de género pero en estos últimos años se han situado en una posición más favorable que las universidades públicas, donde la presencia fe- menina alcanza al $36,74 \%$. Ello podría deberse a que las universidades privadas centran mayormente su enseñanza en áreas de Ciencias Sociales y Sanitarias.

Respecto al colectivo de Personal de Administración y Servicios (PAS), el porcentaje de mujeres y hombres en las universidades públicas y privadas, en cambio, incrementó sus diferencias en los últimos años, en favor de las primeras. Así, las mujeres son el $58,01 \%$ y el $62,56 \%$ de los miembros del PAS de las universidades públicas y privadas, respectivamente, en el curso 2008-2009.

En el Gráfico 1 se presenta la conocida gráfica de tijeras, que compara la distribución de mujeres y hombres en los distintos niveles académicos de las universidades públicas españolas. Esta gráfica no incluye el seguimiento de cohortes, es decir, el seguimiento de las mujeres que comenzaron sus estudios universitarios y que han llegado a ser profesoras titulares o catedráticas, pero sí permite visibilizar de manera muy clara el desequilibrio de género de las universidades españolas. De este modo, se puede comprobar que las mujeres estudiantes son 


\section{Tabla 3}

Representación demográfica por género en los tres sectores laborales universitarios (en \%)

\begin{tabular}{|c|c|c|c|c|c|c|c|c|}
\hline \multirow{3}{*}{$\begin{array}{l}\text { Sectores } \\
\text { Universita- } \\
\text { rios }\end{array}$} & \multicolumn{4}{|c|}{ Curso 1996-1997 } & \multicolumn{4}{|c|}{ Curso 2008-2009 } \\
\hline & \multicolumn{2}{|c|}{$\begin{array}{l}\text { Universidades } \\
\text { Públicas }\end{array}$} & \multicolumn{2}{|c|}{$\begin{array}{l}\text { Universidades } \\
\text { Privadas }\end{array}$} & \multicolumn{2}{|c|}{$\begin{array}{l}\text { Universidades } \\
\text { Públicas }\end{array}$} & \multicolumn{2}{|c|}{$\begin{array}{l}\text { Universidades } \\
\text { Privadas }\end{array}$} \\
\hline & Hombres & Mujeres & Hombres & Mujeres & Hombres & Mujeres & Hombres & Mujeres \\
\hline $\begin{array}{l}\text { Personal } \\
\text { Docente e } \\
\text { Investigador }\end{array}$ & 67,51 & 32,49 & 66,09 & 23,91 & 63,26 & 36,74 & 57,79 & 42,21 \\
\hline $\begin{array}{c}\text { Personal de } \\
\text { Administra- } \\
\text { ción y } \\
\text { Servicios }\end{array}$ & 43,67 & 56,33 & 40,08 & 59,92 & 41,99 & 58,01 & 37,44 & 62,56 \\
\hline
\end{tabular}

Fuente: Elaboración propia a partir de López y Martínez (1999) e INE (1998 y 2010).

\section{Gráfico 1}

Distribución por género de los distintos estadios de la carrera profesional del Personal Docente e Investigador

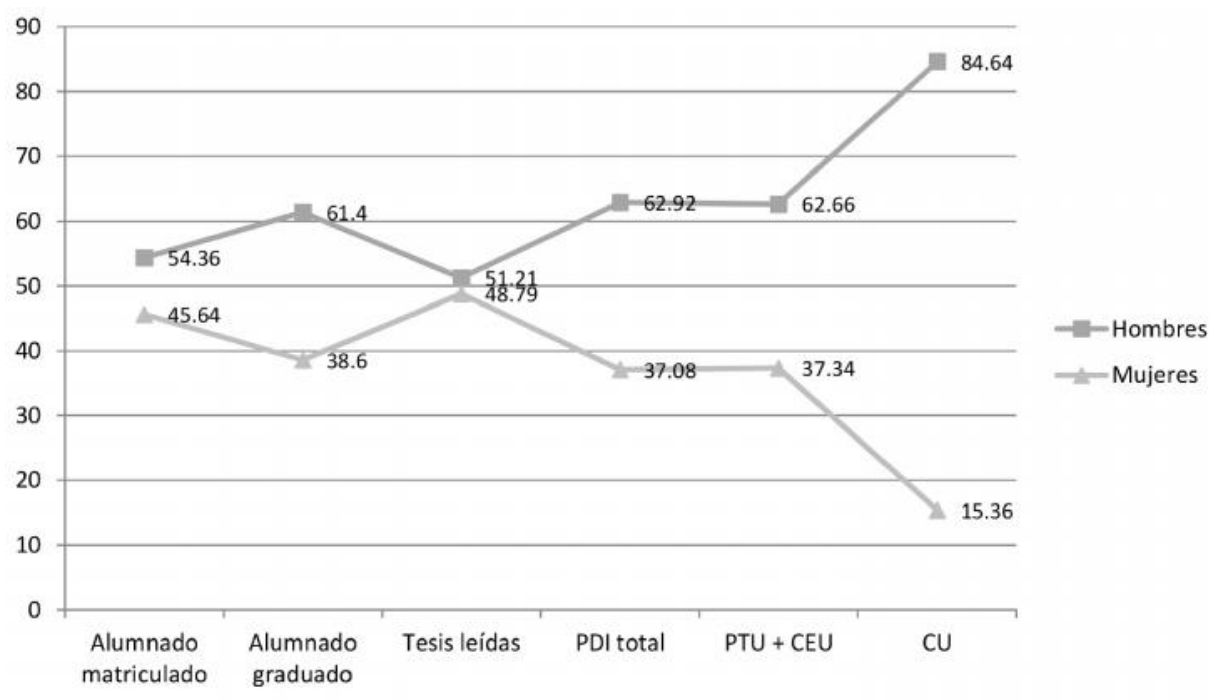

Fuente: Elaboración propia a partir del INE (2011). 
mayoría en las universidades y sus cifras de representación parece que se van igualando en el nivel académico superior, como es el doctorado, pero existe una desigualdad evidente en la carrera profesional como docentes universitarios.

Si bien las diferencias de género en los cuerpos de Profesores Titulares de Universidad y Catedráticos de Escuelas Universitarias (CEU) están próximas a alcanzar una cierta paridad, existe una brecha importante en el cuerpo de Catedráticos de Universidad (CU). No obstante, es preciso destacar la tendencia alcista en cuanto al número de profesoras que alcanzan las categorías académicas más elevadas. Así, en los ocho últimos años (2002-2009), de los que se disponen datos desagregados por categorías laborales en el Instituto Nacional de Estadística, las Profesoras Titulares de Universidad y las Catedráticas de Escuelas Universitarias han pasado del $34,39 \%$ al $37,34 \%$, mientras que las Catedráticas de Universidad han pasado del $12,75 \%$ al $15,36 \%$.

\subsection{La representación política por género en las universidades españolas}

En el presente estudio, la representación política se refiere a los órganos unipersonales y los órganos colegiados más relevantes jerárquicamente en las universidades españolas. En este sentido, los órganos unipersonales seleccionados son los cargos de Rector/a, Vicerrectores, Secretaría General y Gerente, mientras que los órganos colegiados son el Consejo de Gobierno, el Claustro Universitario y el Consejo Social. Según la legislación vigente (Gobierno de España, 2007b) las universidades públicas y privadas deben tener los mismos órganos unipersonales, pero no ocurre así con los órganos colegiados, en los que hay más laxitud en la estructura de dirección de las universidades privadas. Ante esta casuística, el análisis de los datos se concreta en los órganos unipersonales tanto de universidades públicas como privadas y sólo en los órganos colegiados de las universidades públicas.

Así pues, respecto a los órganos unipersonales de gobierno (Tabla 4), las universidades alcanzan en el curso 2008-2009 una presencia paritaria por género en las Secretarías Generales ( $44,11 \%$ de mujeres) y se aproximan a alcanzarla en los Vicerrectorados (37,08\% de mujeres). En cambio, los cargos de Rector y Gerente son ocupados mayoritariamente por hombres. Hay que puntualizar que las universidades privadas ofrecen una mayor representación femenina en estos dos últimos órganos unipersonales, mientras las universidades públicas destacan en los otros dos órganos. Esto es reflejo de la mayor "lucha en solitario" de la mujer en la empresa privada frente a la existencia de un mayor apoyo en la administración pública.

Las diferencias presentadas se suavizan a lo largo del tiempo. Así, hay que destacar una evolución positiva en el tiempo se contempla equipo de gobierno de las universidades en su conjunto, donde la representación femenina supone alrededor de un tercio en 2009, mientras que 12 años atrás, de acuerdo con López y Martínez (1999), tan sólo alcanzaba un 15,5\%.

Por otra parte, los órganos colegiados de las universidades públicas (Consejo de Gobierno, Claustro y Consejo So- 


\section{Tabla 4}

\section{Representación por género en los órganos de gobierno unipersonales de las universidades (Curso 2008-2009)}

\begin{tabular}{ccccccccc}
\hline & \multicolumn{2}{c}{ Rectores } & \multicolumn{2}{c}{$\begin{array}{c}\text { Secretarías } \\
\text { Generales }\end{array}$} & \multicolumn{2}{c}{ Gerentes } & \multicolumn{2}{c}{ Vicerrectores } \\
\cline { 2 - 9 } & Mujeres & Hombres & Mujeres & Hombres & Mujeres & Hombres & Mujeres & Hombres \\
\hline $\begin{array}{c}\text { Universidad } \\
\text { Pública }\end{array}$ & 10,20 & 89,80 & 47,92 & 52,08 & 14,29 & 85,71 & 38,56 & 61,65 \\
$\begin{array}{c}\text { Universidad } \\
\text { Privada }\end{array}$ & 31,82 & 68,18 & 35,00 & 65,00 & 28,57 & 78,57 & 25,81 & 77,42 \\
$\quad$ TOTAL & 16,90 & 83,10 & 44,11 & 55,89 & 17,46 & 82,54 & 37,08 & 62,92 \\
\hline
\end{tabular}

Fuente: Elaboración propia a partir del INE (2011).

cial), muestran también la desigual representación entre mujeres y hombres en los puestos de toma de decisiones. La composición de estos órganos está formada por varios colectivos: PDI, PAS, alumnado y, en su caso, personal externo a la universidad. Este último colectivo aparece exclusivamente en el Consejo Social. La cuota de representación de estos colectivos viene regulada por los Estatutos de las Universidades.

Comparando los dos cursos académicos analizados, la participación de hombres y mujeres apenas varió en los Consejos de Gobierno de las universidades públicas, ascendiendo ligeramente la presencia femenina en los tres colectivos que lo representan (PDI, PAS y alumnado), pero todavía alejada del equilibrio necesario. Agrava la situación que las mujeres estudiantes sigan teniendo una representación tan baja en la toma de decisiones universitaria, a pesar de que en el transcurso de estos 12 años el número de alumnas superó al número de alumnos (Tabla 5).

En lo que concierne al Claustro Universitario, los resultados obtenidos revelan la misma situación observada en el Consejo de Gobierno. A pesar de existir una considerable mayoría de mujeres ocupando los colectivos del PAS $(53,40 \%)$ y del alumnado $(54,36 \%)$ en las universidades durante el curso 2008-2009, esta presencia dominante no se corresponde con su representación en la toma de decisiones. De esta manera, las mujeres que representan al PAS y a los alumnos en el Claustro son un $41 \%$ y un $40 \%$, respectivamente. Estos datos ofrecen un mayor equilibrio que en el caso del Consejo de Gobierno. Sin embargo, los representantes del PDI en el Claustro ofrecen un claro desajuste, contando con una presencia femenina limitada al $28,35 \%$. Esto puede ser explicado por las grandes diferencias existentes en el cuerpo de Catedráticos de Universidad (Tabla 6).

Si se comparan los datos anteriores con los del curso 1996-1997, la representación de las mujeres en el colectivo del PAS en el Claustro disminuyó casi en seis puntos porcentuales y la del colectivo del alumnado descendió también en tres puntos. Aunque su presencia está aún dentro de las cuotas de representa- 


\section{Tabla 5}

\section{Participación de los sectores y géneros en los Consejos de Gobierno} de las universidades

\begin{tabular}{lcccc}
\hline \multirow{2}{*}{ Sectores } & \multicolumn{2}{c}{ Curso académico 1996-1997 } & \multicolumn{2}{c}{ Curso académico 2008-2009 } \\
\cline { 2 - 5 } & Hombres (\%) & Mujeres (\%) & Hombres (\%) & Mujeres (\%) \\
\hline $\begin{array}{l}\text { Personal Docente } \\
\text { e Investigador }\end{array}$ & 73,79 & 26,21 & 71,43 & 28,28 \\
Alumnado & 70,99 & 29,01 & & \\
Personal de & 64,06 & 35,94 & 73,40 & 29,56 \\
Administración y & & & 63,11 & 37,70 \\
Servicio & & & & \\
TOTAL & 79,51 & 20,49 & 71,13 & 28,97 \\
\hline
\end{tabular}

Fuente: Elaboración propia a partir de López y Martínez (1999).

\section{Tabla 6}

\section{Participación de los sectores y géneros en los Claustros de las universidades}

\begin{tabular}{lcccc}
\hline \multirow{2}{*}{ Sectores } & \multicolumn{2}{c}{ Curso académico 1996-1997 } & \multicolumn{2}{c}{ Curso académico 2008-2009 } \\
\cline { 2 - 5 } & Hombres (\%) & Mujeres (\%) & Hombres (\%) & Mujeres (\%) \\
\hline $\begin{array}{l}\text { Personal Docente } \\
\text { e Investigador }\end{array}$ & 74,34 & 25,66 & 68,81 & 31,19 \\
$\begin{array}{l}\text { Personal de } \\
\text { Administración }\end{array}$ & 53,18 & 46,82 & 58,89 & 41,11 \\
y Servicio & & & & \\
Alumnado & 56,92 & 43,08 & 59,96 & 40,04 \\
TOTAL & 67,56 & 33,44 & 65,74 & 34,26 \\
\hline
\end{tabular}

Fuente: Elaboración propia a partir de López y Martínez (1999).

ción equilibrada, es significativo que haya descendido, teniendo en cuenta que las cifras de alumnas matriculadas y de trabajadoras del PAS superan a las del género masculino. Por el contrario, las mujeres que pertenecen al PDI y que forman parte del Claustro han aumentado su presencia con respecto a los hombres en algo más de cinco puntos porcentuales, ocupando actualmente el $31,19 \%$, pero esto no se corresponde con el mayor aumento experimentado en el número de profesoras universitarias en los últimos años. Es decir, las mujeres aumentan su participación en el Claustro en un $17,76 \%$, mientras que aumentan su participación en el PDI en un $26,91 \%$.

Por último, en la composición del Consejo Social del curso 2008-2009 (Tabla 7), se comprueba que es el órgano decisorio con un mayor desequilibrio de representación entre mujeres y hombres en los cuatro colectivos representados (PDI, PAS, alumnado y personal externo a la 


\section{Tabla 7}

\section{Participación de los sectores y géneros en los Consejos Sociales de las universidades}

\begin{tabular}{lcccc}
\hline \multirow{2}{*}{ Sectores } & \multicolumn{2}{c}{ Curso académico 1996-1997 } & \multicolumn{2}{c}{ Curso académico 2008-2009 } \\
\cline { 2 - 5 } & Hombres (\%) & Mujeres (\%) & Hombres (\%) & Mujeres (\%) \\
\hline $\begin{array}{l}\text { Personal Docente } \\
\text { e Investigador }\end{array}$ & & 76,22 & 23,78 \\
$\begin{array}{l}\text { Personal de } \\
\text { Administración }\end{array}$ & 79,35 & 20,65 & 73,33 & 28,89 \\
y Servicio & & & & \\
Alumnado & & & 76,60 & 23,40 \\
Personal Externo & 87,03 & 12,97 & 77,80 & 21,95 \\
TOTAL & 83,96 & 16,04 & 77,40 & 22,60 \\
\hline
\end{tabular}

Fuente: Elaboración propia a partir de López y Martínez (1999).

universidad). La presencia femenina es inferior al $30 \%$ en los cuatro colectivos citados. De este modo, las mujeres ocupan el $23,78 \%$ de la representación del PDI, el $28,89 \%$ de la representación del PAS, el $23,40 \%$ de la del alumnado y el $21,95 \%$ de la del personal externo.

Con los datos disponibles del curso 1996-1997, podemos apreciar el aumento de la presencia femenina en los miembros pertenecientes a los colectivos universitarios (PDI, PAS y alumnado), que alcanza en su conjunto un $20,65 \%$, así como en los miembros pertenecientes a ámbitos externos a las universidades, donde las mujeres suponían un escaso $12,95 \%$ hace 12 años.

\section{Conclusiones}

Las universidades españolas han sido testigo del acceso de las mujeres al espacio público, viendo su presencia creciente en todas las disciplinas y llegando a alcanzar cifras de matriculación mayores que las de la presencia masculina. Con este cambio, a simple vista parece que se está ante una igualdad de oportunidades y una presencia equilibrada entre mujeres y hombres en las universidades. Sin embargo, tanto en los datos europeos aportados por el Informe ETAN (2000), como en España, a raíz de los resultados de este estudio, en consonancia con otros estudios anteriores, las mujeres se enfrentan a una serie de barreras visibles e invisibles que las siguen manteniendo en una posición de desigualdad vinculada aún a roles y valores tradicionales sobre los géneros, aunque se atisba alguna mejoría. Estas barreras podrían ser objeto de análisis en futuras investigaciones.

En el presente trabajo se realizó un diagnóstico de la situación de las universidades españolas desde una perspectiva de género. Se llevó a cabo un análisis longitudinal comparando datos de dos cursos académicos (1996-1997 y 20082009), en el que se profundiza en la presencia de mujeres y hombres en los colectivos universitarios (PDI, PAS y alumnado), así como en la participación de 
ambos géneros en los órganos de gobierno universitarios, tanto unipersonales como colegiados.

A la luz de la normativa vigente sobre democracia paritaria, las universidades tienen un largo camino que recorrer si se pretende alcanzar la igualdad real en la composición de sus órganos de gobierno. Como se citó al inicio de este artículo, esta representación será equilibrada democráticamente cuando incluya los intereses de toda la sociedad, y no sólo de "una parte de ella". Los niveles jerárquicos del mundo académico actual son espacios casi exclusivamente masculinos, produciendo y reproduciendo continuamente una ciencia de carácter androcéntrico que, aunque va abriendo sus puertas a la presencia femenina, este acceso está siendo paralizado por una multitud de barreras visibles e invisibles.

En líneas generales, se observó que la presencia femenina ha aumentado en el número de alumnas matriculadas en primer y segundo ciclos universitarios, constituyendo más de la mitad del alumnado en las universidades públicas y privadas. Asimismo, el número de alumnas de tercer ciclo ha crecido ligeramente respecto al de los alumnos, siendo un hecho significativo el gran aumento de las tesis disertadas por parte de mujeres, que han logrado así alcanzar su justo equilibrio. En estos últimos años, ha incrementado también el porcentaje de docentes femeninas, aunque todavía se mantienen a cierta distancia del equilibrio de género. Esta aparente tendencia a la igualdad formal en el acceso discente y docente a la universidad esconde una doble segregación que sigue manteniendo a las mujeres en situaciones de desigualdad ante los hombres, concluyendo por ello que existe una segregación horizontal y una segregación vertical.

Por un lado, se observa una segregación horizontal en cuanto a que las mujeres siguen concentradas en las ramas de enseñanza de Ciencias de la Salud, Humanidades y Ciencias Sociales, vinculadas al desempeño de los roles que tradicionalmente se les asignaban (los roles de atención y servicios sociales). Por otra parte, existe una segregación vertical al darse una desigualdad entre mujeres y hombres en los distintos niveles de responsabilidad en la jerarquía ocupacional, tanto en las categorías académicas del personal docente como en los órganos de gobierno que ocupan.

Hay que precisar que las cifras de representación por género en los órganos de gobierno, en general, apenas han variado. La presencia femenina ha aumentado ligeramente en la mayoría de cargos ocupados, tanto unipersonales como colegiados, pero sigue siendo escasa al igual que hace una década. Incluso, hay casos en los que ha disminuido la presencia femenina, como ocurre con la representación del PAS y del alumnado en el Claustro universitario. Este hecho es muy significativo y contradictorio si se considera que estos dos colectivos poseen mayores porcentajes de mujeres, incrementándose en los últimos años, y también si se entiende que el Claustro es el máximo órgano representativo de la comunidad universitaria.

La llegada de mujeres a los puestos de responsabilidad en la comunidad universitaria y el aprovechamiento de su potencial requieren importantes cambios en la cultura organizativa de esta institu- 
ción y la aplicación de medidas efectivas para hacer posible la ruptura del techo de cristal. Es necesario seguir trabajando en medidas de conciliación, introducir nuevos valores de liderazgo, modificar en los Estatutos universitarios, las leyes electorales $u$ otras modificaciones que promuevan la representación de las mujeres en los órganos gerenciales, difundir nuevos modelos de paternidad y organización familiar y, sobre todo, transformar las relaciones de poder entre los géneros, creando un espacio simbólico donde mujeres y hombres tengan las mismas oportunidades reales de participar en igualdad en una sociedad democrática.

La educación es uno de los ámbitos para promover la participación de las mujeres en el espacio público y para fomentar su representación en los órganos de decisión de las instituciones. Sin embargo, como en todos los ámbitos de la vida, cuando en una sociedad persiste la transmisión de valores y actitudes tradicionales relacionadas con estereotipos sexistas, aunque el sistema educativo sea formalmente igualitario en cuanto a oportunidades entre mujeres y hombres, esta igualdad formal nunca podrá garantizar la igualdad real.

No cabe duda que la representación femenina en los órganos de gobierno universitarios resulta todavía insuficiente, de acuerdo con las exigencias legislativas de una presencia equilibrada. Los resultados reflejan esta situación de posible persistencia de estereotipos y actitudes sexistas, a pesar de la presencia mayoritaria y creciente de las mujeres en el sistema educativo superior. Una clara muestra de ello es la baja representación de las mujeres estudiantes en los órganos de go- bierno de las universidades públicas. Debe alertar que, desde 1996, el porcentaje de alumnas sea prácticamente igual, cuando no se reduce, en los órganos de decisión universitarios. Por tanto, el techo de cristal no se encuentra únicamente en los factores propios de la carrera profesi onal, sino que hay que buscarlo en sus raíces, es decir, en las etapas previas a la iniciación de dicha carrera.

No obstante, una posible justificación puede ser que la mujeres, en general, estén más presionadas que los hombres en su carrera académica y profesional, y su mayor necesidad de competir e igualarse al género masculino les obligue a concentrar sus esfuerzos personales, de manera que deje de lado otras ocupaciones consideradas secundarias, entre las que puede entenderse su participación en la gerencia universitaria. Para investigaciones futuras, sería interesante analizar en profundidad las razones y circunstancias por las que las mujeres no están ocupando actualmente una mayor representación gerencial en las universidades, tanto por parte del alumnado como por parte del personal universitario.

Como dato alentador, la presencia de la mujer en el cuerpo de Profesores Titulares de Universidad ha evolucionado favorablemente, lo que puede dar indicios de un mayor equilibrio de poder en las universidades en un futuro próximo, disipando las diferencias que existen especialmente en las categorías académicas de más alto rango. Esta evolución positiva se afianza probablemente si observamos los profesores doctores laborales o no funcionarios, que suponen el grupo de docentes universitarios más joven y prometedor, esto es, con mayores expec- 
tativas de consolidar su carrera docente. De este modo, las profesoras Contratadas Doctoras y las profesoras Ayudantes Doctoras alcanzan ya prácticamente la mitad en sus categorías respectivas en el curso 2008-2009. Esto debería tener una consecuencia directa en la reducción de las diferencias de género en las categorías docentes más elevadas en la universidad en un futuro muy próximo, favoreciendo la democracia paritaria.

En este sentido, se considera que el nuevo modelo de promoción del profesorado universitario, implantado ya iniciado este siglo XXI, va a contribuir a democratizar la carrera docente $y$, con ello, se va a generar una mayor igualdad de oportunidades entre mujeres y hombres. Por ello, y como implicaciones para la gerencia universitaria, el suelo de cemento en la carrera profesional se debe seguir combatiendo con propuestas de políticas familiares y laborales que eliminen los factores de desequilibrio entre los distintos niveles académicos y que facilite a la mujer la llegada a los puestos de toma de decisiones universitarias.

Es posible que exista un estilo femenino de gestión y liderazgo que haya que considerar en mayor profundidad para intentar reducir las diferencias de género en los órganos de gobierno universitarios. En este sentido, el poder de las organizaciones, en general, y de las instituciones universitarias, en particular, ha estado definido por el género masculino. De este modo, los pactos, las alianzas y las redes informales y profesionales se han establecido preferentemente entre hombres y muy escasamente entre mujeres en estas organizaciones a lo largo de varias generaciones. En cambio, las mujeres están en un proceso de consolidación laboral si tenemos en cuenta que constituyen casi una primera generación de docentes e investigadoras universitarias, entendido en un sentido amplio, es decir, considerando que las mujeres se han incorporado extensamente al mundo laboral en las últimas décadas y que ya alcanzan una masa crítica entre el profesorado universitario español.

En definitiva, los resultados obtenidos pueden revelar una mayor ineficiencia y una menor calidad en la toma de decisiones gerenciales en las universidades, dado que todavía existe un claro desequilibrio de género en los cargos de gestión universitaria ocupados por hombres y mujeres. Esta falta de aprovechamiento de todo el potencial de conocimientos académicos y de gestión del personal de estas instituciones conlleva una pérdida de competitividad en el ámbito universitario nacional e internacional. Por tanto, tratar de corregir este desequilibrio no es tan sólo una cuestión de justicia e igualdad social.

Para futuras investigaciones, además de las recomendaciones ya indicadas, sería interesante también realizar estudios similares en otras universidades, tanto nacionales como internacionales, tratando de comparar sus datos en función de criterios como la antigüedad, o bien, el carácter público o privado de estas instituciones e, incluso, las diferencias en los estilos de dirección entre hombres y mujeres. Del mismo modo, es preciso seguir analizando en qué medida va mejorando la igualdad de género en la gerencia universitaria a lo largo del tiempo. 


\section{Referencias Bibliográficas}

Escolano, Esther (2006). Discriminación en un medio meritocrático: las profesoras en la universidad española. Revista Mexicana de Sociología, Vol. 68, № 2, pp. 231-263.

European Technology Assessment Network (ETAN) (2000). Science policies in the European Union: Promoting excellence through mainstreaming gender equality. ETAN Expert Working Group on Women and Science. European Commission, Bruselas.

García, Teresa (2007). La endogamia profesional en el ámbito del magisterio docente en España. Revista Internacional de Sociología, Vol. 65, № 48, pp. 147-171.

García de León, Maria Antonia, García de Cortázar, María Luisa (coords.) (2001). Las Académicas. Profesorado universitario y género. Instituto de la Mujer, Madrid.

Gobierno de España (2007a). Ley Orgánica $3 / 2007$, de 22 de marzo, para la igualdad efectiva de mujeres y hombres. Boletín Oficial del Estado, 71, pp. 12611-12645.

Gobierno de España (2007b). Ley Orgánica $4 / 2007$ por la que se modifica la Ley 6/2001, de 21 de diciembre, de Universidades. Boletín Oficial del Estado, 89, pp. 16241-16260.

González Moreno, Maria Cristina (2004). El Género: un enfoque transversal en las políticas de salud del Estado Venezolano. Revista Venezolana de Gerencia, Vol. 9, № 27, pp. 395-413.

Hernández, Azucena; Sánchez, Maria Cruz y García-Valcárcel, Ana (2005). Igualdad de oportunidades en el ejercicio de la función docente universitaria: situación de hombres y mujeres en la universidad del siglo XXI. Revista Educación y Pedagogía, Vol. 16, N . 40, pp. 91-114.
Ibáñez, Marta (2010). Al otro lado de la segregación ocupacional por sexo. Hombres en ocupaciones femeninas y mujeres en ocupaciones masculinas. Revista Internacional de Sociología, Vol. 68, No 1, pp. 145-164.

Instituto Nacional de Estadística (1999). Estadística de la Enseñanza Superior en España. Curso 1996-1997. INE, Madrid.

Instituto Nacional de Estadística (2011). Estadística de Enseñanza Universitaria. Curso 2008-09. INE, Madrid.

Iranzo, Consuelo y Richter, Jacqueline (2000). El espacio femenino en el mundo del trabajo formal. Revista Venezolana de Gerencia, Vol. 7, № 20, pp. 509-535.

López, Jesús Manuel y Martínez, María del Rocío (1999). Estudio de la composición orgánica y del género en la gestión universitaria. En Actas del XIII Congreso Nacional y IX Congreso Hispano-Francés (pp. 167-173). AEDEM, Logroño.

Martínez, María del Rocío; López, Jesús Manuel y Díaz, María del Carmen (2010). Estudio de género en los órganos decisorios de la Universidad. En Actas del II Congreso Universitario (Nacional) de Investigación y Género (pp. 1121). Universidad de Sevilla, Sevilla.

Ministerio de Trabajo y Asuntos Sociales (2007). Plan Estratégico de Igualdad de Oportunidades 2008-2011. Gobierno de España, Madrid.

Rico, Margarita y Gómez-Limón, José Antonio (2011). Propuesta metodológica para la construcción de indicadores sintéticos de igualdad de género. El caso del medio rural de Castilla y León. Revista Internacional de Sociología, Vol. $69, \mathrm{~N}^{\circ}$ 1, pp. 253-286.

Rodríguez, Adelina (2006). Educación Superior y segregación de género: órganos unipersonales de la Universidad de León. Cuestiones de Género: de la igualdad y la diferencia, Vol. 1, pp. 137-153. 
Tómas, Marina y Guillamón, Cristina (2009). Las barreras y los obstáculos en el acceso de las profesoras universitarias a los cargos de gestión académica. Revista de Educación, Vol. 350, pp. 253-275.

Tómas, Marina; Durán, María del Mar y Guillamón, Cristina (2009). La implicación de las profesoras en la gestión universita- ria. Revista Interuniversitaria de Pedagogía Social, Vol. 16, pp.95-104.

White, Kate; Riordan, Sarah; Ozkanli, Ozlem y Neale, Jenny (2010). Cross Cultural perspectives of gender and management in universities. South African Journal of Higher Education, Vol. $24, N^{\circ} .4$, pp. 646-660. 